\title{
Protocolo de tratamento do mieloma múltiplo: serviço de hematologia Hospital Universitário Walter Cantídio, abril - 2019
}

\section{Multiple myeloma treatment protocol: Walter Cantídio University Hospital hematology service, april - 2019}

Jacqueline Holanda de Sousa ${ }^{1}$. Herivaldo Ferreira da Silva² ${ }^{2}$ Livia Andrade Gurgel ${ }^{3}$. João Paulo de Vasconcelos Leitão ${ }^{4}$. Beatriz Stela Gomes de Sousa Pitombeira Araújo ${ }^{4}$. Karine Sampaio Nunes Barroso ${ }^{4}$. Thaisa Marjore Menezes Viana ${ }^{3}$. Romélia Pinheiro Gonçalves Lemes². Fernando Barroso Duarte ${ }^{2,3}$.

1 Secretaria de Saúde do Estado do Ceará (SESA), Fortaleza, Ceará, Brasil. 2 Universidade Federal do Ceará (UFC), Fortaleza, Ceará, Brasil. 3 Hospital Universitário Walter Cantídio (HUWC), Fortaleza, Ceará, Brasil. 4 Empresa Brasileira de Serviços Hospitalares (EBSERH), Fortaleza, Ceará, Brasil.

\section{RESUMO}

O Mieloma Múltiplo (MM) é uma neoplasia hematológica caracterizada pela infiltração de medula óssea por plasmócitos clonais (malignos) e pela produção de uma proteína monoclonal (PM). A doença permanece incurável, embora, nos últimos anos, com o advento de novos tratamentos, tenha-se observado melhoras progressivas nas taxas e duração da remissão, traduzindo-se por um aumento na sobrevida dos pacientes acometidos. Portanto, esse trabalho tem como objetivo descrever o protocolo de tratamento do serviço de hematologia de um hospital universitário.

Palavras-chave: Mieloma múltiplo. Tratamento. Diagnóstico.

\section{ABSTRACT}

Multiple Myeloma (MM) is a hematological neoplasm characterized by bone marrow infiltration by clonal (malignant) plasma cells and the production of a monoclonal protein (MP). The disease remains incurable, although in recent years, with the advent of new treatments, progressive improvements in rates and duration of remission have been observed, resulting in a significant survival increment for affected patients. Therefore, this paper aims to describe the treatment protocol of the hematology service of a university hospital.

Keywords: Multiple myeloma. Treatment. Diagnosis.

Autor correspondente: Romélia Pinheiro Gonçalves Lemes, Rua Pastor Samuel Munguba, 1210, Rodolfo Teófilo, Fortaleza, Ceará. CEP: 60430372. Telefone: +55 85 3366-8264. E-mail: romeliagonçalves@gmail.com

Conflito de interesses: Não há qualquer conflito de interesses por parte de qualquer um dos autores.

Recebido em: 28 Ago 2019; Revisado em: 20 Nov 2019; Aceito em: 20 Nov 2019. 


\section{INTRODUÇÃO}

O Mieloma Múltiplo (MM) é uma neoplasia hematológica caracterizada pela infiltração da medula óssea por plasmócitos clonais (malignos) e pela produção de uma Proteína Monoclonal (PM), levando à lesão de órgãos alvo: lesões osteolíticas, anemia, insuficiência renal ou hipercalcemia. ${ }^{1,2}$

A doença permanece incurável, embora, nos últimos anos, com o advento de várias novas drogas e do transplante autólogo de células tronco hematopoiéticas (TCTH), tenha-se observado melhoras progressivas nas taxas e duração da remissão, traduzindo-se por um aumento significativo na sobrevida dos pacientes acometidos. ${ }^{3-8}$

\section{DIAGNÓSTICO}

São critérios obrigatórios para o diagnóstico de MM: ${ }^{9}$

1. $10 \%$ ou mais plasmócitos clonais na medula óssea ou biópsia de um plasmocitoma ósseo ou extramedular (critério obrigatório);

2. Pelo menos um evento definidor da doença ou biomarcador de malignidade:

\subsection{Evento definidor da doença:}

2.1.1 Hipercalcemia (Cálcio > 10,5mg/dl);

2.1.2 Insuficiência renal (clearance de creatinina $<40 \mathrm{ml} /$ $\mathrm{m}^{2} / \mathrm{min}$ ou creatinina $>2,0 \mathrm{mg} / \mathrm{dl}$ );

2.1.3 Anemia (hemoglobina $<10 \mathrm{~g} / \mathrm{dl}$ );

2.1.4 Uma ou mais lesões osteolíticas detectadas em Rx do esqueleto, tomografia computadorizada (TC) ou PET$\mathrm{TC}$;

2.2. Biomarcadores de malignidade (conferem o diagnóstico de MM mesmo na ausência de evento definidor da doença):

$3 \geq 60 \%$ de plasmócitos clonais na medula óssea;

4 Taxa de cadeias leves livres envolvidas/não envolvidas $\geq$ 100 (a cadeia leve envolvida necessita ser $\geq 100 \mathrm{mg} / \mathrm{L}$ );

5 Mais de uma lesão focal $\geq 5 \mathrm{~mm}$ em exame de ressonância magnética nuclear.

\section{ESTADIAMENTO}

Esquema de Salmon-Durie ${ }^{10}$

\section{Estádio I - massa celular baixa:}

Todos os itens a seguir presentes:

- Nas radiografias, estrutura óssea normal ou apenas plasmocitoma ósseo solitário;

- Valor de hemoglobina $>10 \mathrm{~g} / \mathrm{dl}$;

- Valor de cálcio sérico normal ou $<10,5 \mathrm{mg} / \mathrm{dl}$;
- Baixa taxa de produção de componente $\mathrm{M}$ com valor de $\operatorname{IgG}<5 \mathrm{~g} / \mathrm{dl}$ e de $\operatorname{IgA}<3 \mathrm{~g} / \mathrm{dl}$;

- Proteína de Bence-Jones $<4 \mathrm{~g} / 24$ horas.

Estádio II - massa celular intermediária:

Não atende aos critérios de estádio I nem de estádio III.

\section{Estádio III - massa celular elevada:}

Um ou mais dos seguintes itens presentes:

- Valor de hemoglobina $<8,5 \mathrm{~g} / \mathrm{dl}$;

- Valor de cálcio sérico > $12 \mathrm{mg} / \mathrm{dl}$;

- Lesões ósseas líticas avançadas;

- Alta taxa de produção de componente M com valor de $\operatorname{IgG}>7 \mathrm{~g} / \mathrm{dl}$ e de $\operatorname{IgA}>5 \mathrm{~g} / \mathrm{dl}$;

- Proteína de Bence-Jones $>12 \mathrm{~g} / 24$ horas.

Subclassificação A: Creatinina sérica $\leq 2,0 \mathrm{mg} / \mathrm{dl}$.

Subclassificação B: Creatinina sérica $>2,0 \mathrm{mg} / \mathrm{dl}$.

Quadro 1. Esquema ISS (International Staging System). ${ }^{11}$

\begin{tabular}{|l|l|l|}
\hline & Concentração sérica & Sobrevida em meses \\
\hline Estágio I & $\begin{array}{l}\beta 2 \text { microglobulina } \leq 3,5 \mathrm{mg} / \mathrm{L} \\
\text { e albumina } \geq 3,5 \mathrm{mg} / \mathrm{dL}\end{array}$ & 62 \\
\hline Estágio II & $\begin{array}{l}\beta 2 \text { microglobulina }<3,5 \mathrm{mg} / \mathrm{L} \\
\text { e albumina }<3,5 \mathrm{mg} / \mathrm{dL}\end{array}$ & 44 \\
\hline Estágio III & $\beta 2$ microglobulina $>5,5 \mathrm{mg} / \mathrm{L}$ & 30 \\
\hline
\end{tabular}

\section{Esquema ISS revisado: ${ }^{12}$}

\section{Estágio I:}

- $\quad$ Albumina $\geq 3,5 \mathrm{mg} / \mathrm{dL}$;

- B2microglobulina $<3,5 \mathrm{mg} / \mathrm{L}$;

- Ausência de citogenética de alto risco;

- DHL normal.

\section{Estágio II:}

- $\quad$ Sem características do Estágio I ou III.

\section{Estágio III:}

- $\quad$ B2microglobulina $>5,5 \mathrm{mg} / \mathrm{L}$;

- Citogenética de alto risco $[\mathrm{t}(4 ; 16), \mathrm{t}(14 ; 16)$ ou del $(17 \mathrm{p})]$ ou DHL elevada.

\section{EXAMES COMPLEMENTARES}

\section{Sangue periférico:}

- Hemograma;

- VHS;

- Glicemia;

- Ureia, creatinina;

- Sódio, potássio, cálcio, magnésio, fósforo; 
- $\quad$ TGO, TGP, GGT, fosfatase alcalina, DHL;

- Bilirrubinas totais, direta e indireta;

- Dosagem das imunoglobulinas: IgG, IgM, IgA;

- Eletroforese de proteínas séricas;

- Imunofixação de proteínas séricas;

- Dosagem de cadeias leves livres;

- B2-microglobulina;

- Sorologias para hepatite B, hepatite C, HIV 1 e 2, HTLV 1 e 2 , Chagas.

\section{Urina:}

- Sumário de urina;

- Coleta de urina de 24 horas para proteinúria;

- Eletroforese de proteínas urinárias.

\section{Medula óssea:}

- Mielograma;

- Imunofenotipagem por citometria de fluxo com quantificação dos plasmócitos clonais;

- Biópsia óssea com imunohistoquímica (determinação da clonalidade e quantificação dos plasmócitos).

\section{Imagem:}

- Inventário ósseo;

- Tomografia computadorizada de corpo inteiro;

- Ressonância magnética nuclear;

- $\quad$ PET-CT;

- Ecocardiograma.

Quadro 2. Critérios para avaliar resposta ao tratamento. ${ }^{13}$

\begin{tabular}{|c|c|}
\hline Categoria de resposta & Critério de resposta \\
\hline Resposta Completa Estrita (sCR) & $\begin{array}{l}\text { Razão das cadeias leves livres normal } \\
\text { Ausência de células clonais na medula óssea por imunofenotipagem } \\
\text { por citometria de fluxo }\end{array}$ \\
\hline Resposta Completa (CR) & $\begin{array}{l}\text { Imunofixação negativa no sangue e urina } \\
\text { Desaparecimento de plasmocitomas }<5 \% \text { plasmócitos na medula } \\
\text { Óssea }\end{array}$ \\
\hline Resposta Parcial Muito Boa (VGPR) & $\begin{array}{l}\text { Proteína monoclonal sérica e urinária detectável por imunofixação mas } \\
\text { não por eletroforese } \\
\text { Redução da proteína monoclonal sérica em } 90 \% \\
\text { Proteína monoclonal urinária menor que } 100 \mathrm{mg} / 24 \text { horas }\end{array}$ \\
\hline Resposta Parcial (PR) & $\begin{array}{l}\text { Redução }>50 \% \text { na proteína monoclonal sérica e redução na proteína } \\
\text { monoclonal urinária }>90 \% \text { ou }<200 \mathrm{mg} / 24 \text { horas } \\
\text { Redução } \geq 50 \% \text { na diferença entre cadeias leves envolvidas e não } \\
\text { envolvidas } \\
\text { Redução } \geq 50 \% \text { nos plasmócitos na medula óssea (nível basal } \geq 30 \% \text { ) } \\
\text { Redução } \geq 50 \% \text { no tamanho de qualquer plasmocitoma pré-existente }\end{array}$ \\
\hline Doença estável & Não preenche critério para CR, VGPR, PR ou doença progressiva \\
\hline Doença progressiva & 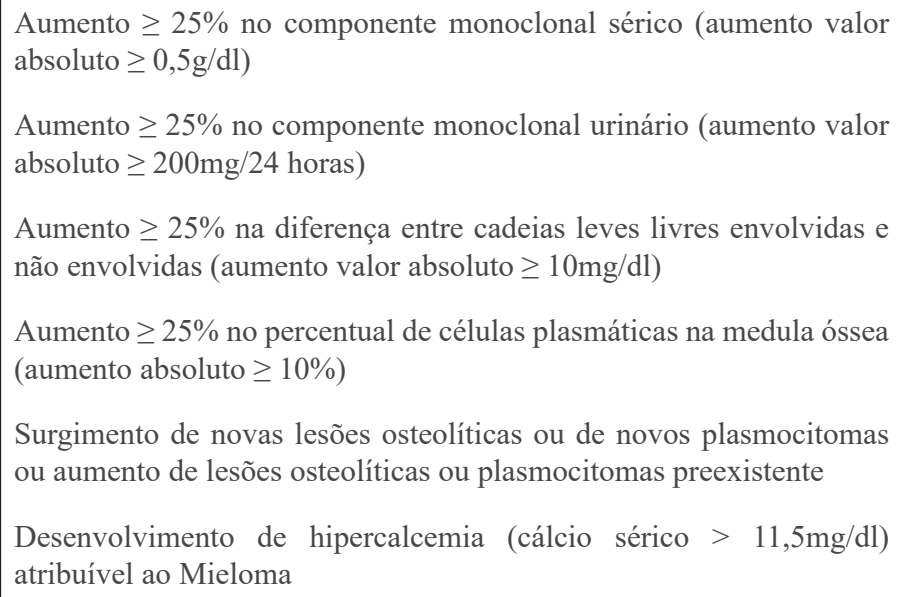 \\
\hline
\end{tabular}




\section{TRATAMENTO PRIMEIRA LINHA}

1. Idade inferior a 75 anos e elegíveis ao transplante autólogo de células tronco hematopoiéticas (TCTH):

Quadro 3. CTD por 4 a 6 ciclos seguido do TCTH autólogo. ${ }^{14}$

\begin{tabular}{|l|l|l|}
\hline DIA & DROGA & DOSE \\
\hline 1 & $\begin{array}{l}\text { Ciclofosfamida } \\
\text { Dexametasona }\end{array}$ & $\begin{array}{l}250 \mathrm{mg} / \mathrm{m}^{2} \text { IV OU ORAL } \\
40 \mathrm{mg} \text { IV OU ORAL }\end{array}$ \\
\hline 8 & $\begin{array}{l}\text { Ciclofosfamida } \\
\text { Dexametasona }\end{array}$ & $\begin{array}{l}250 \mathrm{mg} / \mathrm{m}^{2} \text { IV OU ORAL } \\
40 \mathrm{mg} \text { IV OU ORAL }\end{array}$ \\
\hline 15 & $\begin{array}{l}\text { Ciclofosfamida } \\
\text { Dexametasona }\end{array}$ & $\begin{array}{l}250 \mathrm{mg} / \mathrm{m}^{2} \text { IV OU ORAL } \\
40 \mathrm{mg} \text { IV OU ORAL }\end{array}$ \\
\hline 28 & Dexametasona & $40 \mathrm{mg}$ IV OU ORAL \\
\hline 1 to 28 & Thalidomide & 100 to $200 \mathrm{mg}$ ORAL \\
\hline
\end{tabular}

2. Idade inferior a 75 anos e elegíveis ao transplante autólogo de células tronco hematopoiéticas, porém, não obtiveram pelo menos resposta parcial após 04 ciclos de CTD:

Quadro 4. VCD por 4 a 6 ciclos seguido do TCTH autólogo. ${ }^{15-18}$

\begin{tabular}{|l|l|l|}
\hline DIA & DROGA & DOSE \\
\hline 1 & Ciclofosfamida & $250 \mathrm{mg} / \mathrm{m}^{2}$ IV OU ORAL \\
& Dexametasona & $40 \mathrm{mg}$ IV OU ORAL \\
& Bortezomibe & $1,5 \mathrm{mg} / \mathrm{m}^{2} \mathrm{SC}$ \\
\hline 8 & Ciclofosfamida & $250 \mathrm{mg} / \mathrm{m}^{2}$ IV OU ORAL \\
& Dexametasona & $40 \mathrm{mg}$ IV OU ORAL \\
& Bortezomibe & $1,5 \mathrm{mg} / \mathrm{m}^{2}$ SC \\
\hline \multirow{2}{*}{15} & Ciclofosfamida & $250 \mathrm{mg} / \mathrm{m}^{2}$ IV OU ORAL \\
& Dexametasona & $40 \mathrm{mg}$ IV OU ORAL \\
& Bortezomibe & $1,5 \mathrm{mg} / \mathrm{m}^{2} \mathrm{SC}$ \\
\hline 28 & Dexametasona & $40 \mathrm{mg}$ IV OU ORAL \\
& Bortezomibe & $1,5 \mathrm{mg} / \mathrm{m}^{2}$ SC \\
\hline
\end{tabular}

3. Primeira linha com idade inferior a 75 anos e elegíveis ao transplante autólogo de células tronco hematopoiéticas com insuficiência renal atribuível ao mieloma (creatinina $>2,0 \mathrm{mg}$ / Dl ou clearance de creatinina $<40 \mathrm{ml})$ :

Quadro 5. VCD por 4 a 6 ciclos seguido do TCTH autólogo. 19,20

\begin{tabular}{|l|l|l|}
\hline DIA & DROGA & DOSE \\
\hline 1 & Ciclofosfamida & $250 \mathrm{mg} / \mathrm{m}^{2}$ IV OU ORAL \\
& Dexametasona & $40 \mathrm{mg} \mathrm{IV} \mathrm{OU} \mathrm{ORAL}$ \\
& Bortezomibe & $1,5 \mathrm{mg} / \mathrm{m}^{2} \mathrm{SC}$ \\
\hline
\end{tabular}

Continua.
Conclusão.

Quadro 5. VCD por 4 a 6 ciclos seguido do TCTH autólogo. ${ }^{19,20}$

\begin{tabular}{|l|l|l|}
\hline DIA & DROGA & DOSE \\
\hline 8 & Ciclofosfamida $250 \mathrm{mg} / \mathrm{m}^{2}$ IV & $250 \mathrm{mg} / \mathrm{m}^{2}$ IV OU ORAL \\
& OU ORAL & $40 \mathrm{mg}$ IV OU ORAL \\
& Dexametasona & $1,5 \mathrm{mg} / \mathrm{m}^{2} \mathrm{SC}$ \\
\hline 15 & Ciclofosfamida & $250 \mathrm{mg} / \mathrm{m}^{2}$ IV OU ORAL \\
& Dexametasona & $40 \mathrm{mg}$ IV OU ORAL \\
& Bortezomibe & $1,5 \mathrm{mg} / \mathrm{m}^{2} \mathrm{SC}$ \\
\hline
\end{tabular}

4. Idade superior a 75 anos ou não elegíveis ao transplante autólogo de células tronco hematopoiéticas:

Quadro 6. MPT por 09 a 12 ciclos. $^{21}$

\begin{tabular}{|l|l|l|}
\hline DIA & DROGA & DOSE \\
\hline $1-4$ & Melfalan & $9 \mathrm{mg} / \mathrm{m}^{2}$ ORAL \\
\hline $1-4$ & Prednisona & $60 \mathrm{mg} / \mathrm{m}^{2}$ ORAL \\
\hline $1-42$ & Talidomida & 100 a $200 \mathrm{mg}$ ORAL \\
\hline
\end{tabular}

Obs.: Apesar do Estudo FIRST ${ }^{22}$ demonstrar superioridade de RD contínuo em relação ao MPT, decidimos manter a disponibilidade deste protocolo tendo em vista os seguintes fatores:

a. Indisponibilidade da LENALIDOMIDA no SUS com necessidade de judicialização para sua obtenção.

b. Ausência de estudo equivalente que utilizasse o CTD como braço comparador ao RD contínuo.

c. Intermitência da disponibilidade de medicações no SUS (passamos por períodos de indisponibilidade do MELFALAN e da CICLOFOSFAMIDA oral).

OU

Quadro 7. CTD por 6 a 09 ciclos. ${ }^{23}$

\begin{tabular}{|l|l|l|}
\hline DIA & DROGA & DOSE \\
\hline 1 & $\begin{array}{l}\text { Ciclofosfamida } \\
\text { Dexametasona }\end{array}$ & $\begin{array}{l}250 \mathrm{mg} / \mathrm{m}^{2} \text { IV OU ORAL } \\
40 \mathrm{mg} \text { IV OU ORAL }\end{array}$ \\
\hline 8 & $\begin{array}{l}\text { Ciclofosfamida } \\
\text { Dexametasona }\end{array}$ & $\begin{array}{l}250 \mathrm{mg} / \mathrm{m}^{2} \text { IV OU ORAL } \\
40 \mathrm{mg} \text { IV OU ORAL }\end{array}$ \\
\hline 15 & $\begin{array}{l}\text { Ciclofosfamida } \\
\text { Dexametasona }\end{array}$ & $40 \mathrm{mg}$ IV OU ORAL \\
\hline 28 & Dexametasona & $40 \mathrm{mg}$ IV OU ORAL OU ORA \\
\hline 1 to 28 & Thalidomide & 100 to $200 \mathrm{mg}$ ORAL \\
\hline
\end{tabular}




\section{TRATAMENTO CONSOLIDAÇÃO}

Pacientes que após o TCTH autólogo obtiveram menos que uma resposta parcial muito boa.

Quadro 8. VCD por 2 a 4 ciclos.

\begin{tabular}{|c|c|c|}
\hline DIA & DROGA & DOSE \\
\hline 1 & $\begin{array}{l}\text { Ciclofosfamida } \\
\text { Dexametasona } \\
\text { Bortezomibe }\end{array}$ & $\begin{array}{l}250 \mathrm{mg} / \mathrm{m}^{2} \mathrm{IV} \text { OU ORAL } \\
40 \mathrm{mg} \text { IV OU ORAL } \\
1,5 \mathrm{mg} / \mathrm{m}^{2} \mathrm{SC}\end{array}$ \\
\hline 8 & $\begin{array}{l}\text { Ciclofosfamida } \\
\text { Dexametasona } \\
\text { Bortezomibe }\end{array}$ & $\begin{array}{l}250 \mathrm{mg} / \mathrm{m}^{2} \text { IV OU ORAL } \\
40 \mathrm{mg} \text { IV OU ORAL } \\
1,5 \mathrm{mg} / \mathrm{m}^{2} \mathrm{SC}\end{array}$ \\
\hline 15 & $\begin{array}{l}\text { Ciclofosfamida } \\
\text { Dexametasona } \\
\text { Bortezomibe }\end{array}$ & $\begin{array}{l}250 \mathrm{mg} / \mathrm{m}^{2} \text { IV OU ORAL } \\
40 \mathrm{mg} \text { IV OU ORAL } \\
1,5 \mathrm{mg} / \mathrm{m}^{2} \mathrm{SC}\end{array}$ \\
\hline 28 & $\begin{array}{l}\text { Dexametasona } \\
\text { Bortezomibe }\end{array}$ & $\begin{array}{l}40 \mathrm{mg} \text { IV OU ORAL } \\
1,5 \mathrm{mg} / \mathrm{m}^{2} \mathrm{SC}\end{array}$ \\
\hline
\end{tabular}

\section{TRATAMENTO MANUTENÇÃO}

1. TALIDOMIDA 50 a $100 \mathrm{mg}$ por dia, ORAL, por 12 meses: ${ }^{23-25}$ Pacientes que após o tratamento completo de primeira linha obtiveram menos que uma resposta parcial muito boa, a menos que haja histórico de toxicidade impeditiva com o uso da TALIDOMIDA (em especial neurotoxicidade): TALIDOMIDA 50 a $100 \mathrm{mg}$ por dia, ORAL, por 12 meses, ${ }^{24-26}$

2. LENALIDOMIDA $15 \mathrm{mg}$ ao dia do D1 ao D21 de um ciclo de $28 \operatorname{dias}^{27}$ de forma contínua até progressão da doença ou toxicidade impeditiva.

\section{TRATAMENTO DOENÇA RECAÍDA OU REFRATÁRIA}

1. Nos casos de recaída mais de um ano após o término do tratamento inicial pode-se repetir o mesmo regime utilizado na primeira linha.

2. Dar preferência aos esquemas que utilizam três drogas em oposição a duas drogas.

3. Adequar o tratamento ao perfil de toxicidade prévia, comorbidades e situação clínica específica de cada paciente.

4. Realizar o necessário ajuste de doses conforme a idade e histórico de toxicidade prévia.

São opções para o tratamento do Mieloma Múltiplo recaído ou refratário:

Dara-VD (Castor) ${ }^{28}$
Quadro 9. Ciclos 1 a 3.

\begin{tabular}{|l|l|l|}
\hline DROGA & DIA & DOSE \\
\hline Daratumumab & D1, D8, D15 & $16 \mathrm{mg} / \mathrm{m}^{2}$ EV \\
\hline Bortezomibe & D1, D8, D15, D22 & $1,5 \mathrm{mg} / \mathrm{m}^{2}$ SC \\
\hline Dexametasona & D1, D2, D8, D9, D15, D16 & $20 \mathrm{mg}$ ORAL \\
\hline
\end{tabular}

Obs.: ciclos de 21 dias.

Quadro 10. Ciclos 4 a 8.

\begin{tabular}{|l|l|l|}
\hline DROGA & DIA & DOSE \\
\hline Daratumumab & D1 & $16 \mathrm{mg} / \mathrm{m}^{2} \mathrm{EV}$ \\
\hline Bortezomibe & D1, D8, D15, D22 & $1,5 \mathrm{mg} / \mathrm{m}^{2}$ SC \\
\hline Dexametasona & D1, D2, D8, D9, D15, D16 & $20 \mathrm{mg}$ ORAL \\
\hline
\end{tabular}

Obs.: Ciclos de 21 dias.

Quadro 11. Ciclos 9 em diante.

\begin{tabular}{|l|l|l|}
\hline DROGA & DIA & DOSE \\
\hline Daratumumab & D1 & $16 \mathrm{mg} / \mathrm{m}^{2} \mathrm{EV}$ \\
\hline
\end{tabular}

Obs.1: Tratamento contínuo até progressão ou toxicidade impeditiva.

Obs.2: O bortezomibe será feito em dose única semanal de $1,5 \mathrm{mg} / \mathrm{m}^{2}$ tendo em vista conveniência do serviço.

\section{Dara-RD (Polux). ${ }^{29}$}

Quadro 12. Ciclos 1 e 2.

\begin{tabular}{|l|l|l|}
\hline DROGA & DIA & DOSE \\
\hline Daratumumab & D1, D8, D15 E D22 & $16 \mathrm{mg} / \mathrm{m}^{2} \mathrm{EV}$ \\
\hline Lenalidomida & D1 A D21 & $25 \mathrm{mg}$ ORAL \\
\hline Dexametasona & $\begin{array}{l}\text { D1, D2, D8, D9, D15, D16, } \\
\text { D22, D23 }\end{array}$ & 20mg ORAL \\
\hline
\end{tabular}

Quadro 13. Ciclos 3 a 6 .

\begin{tabular}{|l|l|l|}
\hline DROGA & DIA & DOSE \\
\hline Daratumumab & D1 e D15 & $16 \mathrm{mg} / \mathrm{m}^{2}$ EV \\
\hline Lenalidomida & D1 A D21 & $25 \mathrm{mg}$ ORAL \\
\hline Dexametasona & $\begin{array}{l}\text { D1, D2, D8, D9, D15, D16, } \\
\text { D22, D23 }\end{array}$ & 20mg ORAL \\
\hline
\end{tabular}

Quadro 14. Ciclos 7 em diante.

\begin{tabular}{|l|l|l|}
\hline DROGA & DIA & DOSE \\
\hline Daratumumab & D1 & $16 \mathrm{mg} / \mathrm{m}^{2}$ EV \\
\hline Lenalidomida & D1 A D21 & $25 \mathrm{mg}$ \\
\hline Dexametasona & $\begin{array}{l}\text { D1, D2, D8, D9, D15, D16, } \\
\text { D22, D23 }\end{array}$ & $20 \mathrm{mg}$ \\
\hline
\end{tabular}

Obs.1: Tratamento contínuo até progressão ou toxicidade impeditiva. Obs.2: Redução da dose de LENALIDOMIDA para $10 \mathrm{mg}$ ao dia se Clearance de Creatinina $\leq 60 \mathrm{ml} / \mathrm{min} / 1,73 \mathrm{~m}^{2}$. 


\section{KRD (Aspire). ${ }^{30,31}$}

Quadro 15. Ciclos 1 a 12.

\begin{tabular}{|l|l|l|}
\hline DROGA & DIA & DOSE \\
\hline Carfilzomibe & $\begin{array}{l}\text { D1, D2, D8, D9, } \\
\text { D15, D16 }\end{array}$ & $\begin{array}{l}20 \mathrm{mg} / \mathrm{m}^{2} \text { EV D1 e D2 } \\
27 \mathrm{mg} / \mathrm{m}^{2} \text { EV demais doses }\end{array}$ \\
\hline Lenalidomida & D1 a D21 & $25 \mathrm{mg}$ ORAL \\
\hline Dexametasona & D1, D8, D15, D22 & $40 \mathrm{mg}$ ORAL ou EV \\
\hline
\end{tabular}

Quadro 16. Ciclos 13 a 18.

\begin{tabular}{|l|l|l|}
\hline DROGA & DIA & DOSE \\
\hline Carfilzomibe & D1, D2, D15, D16 & $27 \mathrm{mg} / \mathrm{m}^{2}$ EV demais doses \\
\hline Lenalidomida & D1 a D21 & 25mg ORAL \\
\hline Dexametasona & D1, D8, D15, D22 & $40 \mathrm{mg}$ ORAL ou EV \\
\hline
\end{tabular}

Quadro 17. Ciclos 19 em diante.

\begin{tabular}{|l|l|l|}
\hline DROGA & DIA & DOSE \\
\hline Lenalidomida & D1 a D21 & 25mg ORAL \\
\hline Dexametasona & D1, D8, D15, D22 & $40 \mathrm{mg}$ ORAL ou EV \\
\hline
\end{tabular}

Obs.: Tratamento contínuo até progressão da doença ou toxicidade impeditiva.

Quadro 18. Ixa-RD (Tourmaline). ${ }^{32}$

\begin{tabular}{|l|l|l|}
\hline DROGA & DIA & DOSE \\
\hline Ixazomibe & D1, D8, D15 & $4 \mathrm{mg}$ ORAL \\
\hline Lenalidomida & D1 a D21 & $25 \mathrm{mg}$ ORAL \\
\hline Dexametasona & D1, D8, D15, D22 & 40mg ORAL \\
\hline
\end{tabular}

Obs.1: Tratamento contínuo até progressão ou toxicidade impeditiva. Obs.2: Dose de LENALIDOMIDA reduzida para 10mg D1 a D21 em pacientes com Clearance de Creatinina $\leq 60 \mathrm{ml} / \mathrm{min} / 1,73 \mathrm{~m}^{2}$.

\section{Elo-RD (Eloquent). ${ }^{33}$}

Quadro 19. Ciclos 1 e 2 .

\begin{tabular}{|l|l|l|}
\hline DROGA & DIA & DOSE \\
\hline Elotuzumab & D1, D8, D15, D22 & 10mg/Kg EV \\
\hline Lenalidomida & D1 a D21 & $25 \mathrm{mg}$ ORAL \\
\hline Dexametasona & D1, D8, D15, D22 & ORAL ou EV \\
\hline
\end{tabular}

Quadro 20. Ciclos 3 em diante.

\begin{tabular}{|l|l|l|}
\hline DROGA & DIA & DOSE \\
\hline Elotuzumab & D1, D15 & $10 \mathrm{mg} / \mathrm{Kg} \mathrm{EV}$ \\
\hline Lenalidomida & D1 a D21 & $25 \mathrm{mg}$ ORAL \\
\hline Dexametasona & D1, D8, D15, D22 & ORAL ou EV \\
\hline
\end{tabular}

Obs.1: Tratamento contínuo até progressão ou toxicidade impeditiva.
Quadro 21. CTD. ${ }^{25,26,34}$

\begin{tabular}{|l|l|l|}
\hline DIA & DROGA & DOSE \\
\hline 1 & $\begin{array}{l}\text { Ciclofosfamida } \\
\text { Dexametasona }\end{array}$ & $\begin{array}{l}250 \mathrm{mg} / \mathrm{m}^{2} \text { IV OU ORAL } \\
40 \mathrm{mg} \text { IV OU ORAL }\end{array}$ \\
\hline 8 & Ciclofosfamida & $250 \mathrm{mg} / \mathrm{m}^{2}$ IV OU ORAL \\
& Dexametasona & $40 \mathrm{mg} \mathrm{IV} \mathrm{OU} \mathrm{ORAL}$ \\
\hline 15 & Ciclofosfamida & $250 \mathrm{mg} / \mathrm{m}^{2}$ IV OU ORAL \\
& Dexametasona & $40 \mathrm{mg} \mathrm{IV} \mathrm{OU} \mathrm{ORAL}$ \\
\hline 28 & Dexametasona & $40 \mathrm{mg}$ IV OU ORAL \\
\hline 1 to 28 & Thalidomide & 100 to $200 \mathrm{mg}$ ORAL \\
\hline
\end{tabular}

Quadro 22. VTD. ${ }^{35,36}$

\begin{tabular}{|l|l|l|}
\hline DIA & DROGA & DOSE \\
\hline 1 & Dexametasona & $40 \mathrm{mg}$ IV OU ORAL \\
& Bortezomibe & $1,5 \mathrm{mg} / \mathrm{m}^{2} \mathrm{SC}$ \\
\hline 8 & Dexametasona & $40 \mathrm{mg} \mathrm{IV} \mathrm{OU} \mathrm{ORAL}$ \\
& Bortezomibe & $1,5 \mathrm{mg} / \mathrm{m}^{2} \mathrm{SC}$ \\
\hline 15 & Dexametasona & $40 \mathrm{mg} \mathrm{IV} \mathrm{OU} \mathrm{ORAL}$ \\
& Bortezomibe & $1,5 \mathrm{mg} / \mathrm{m}^{2} \mathrm{SC}$ \\
\hline 28 & Dexametasona & $40 \mathrm{mg} \mathrm{IV} \mathrm{OU} \mathrm{ORAL}$ \\
& Bortezomibe & $1,5 \mathrm{mg} / \mathrm{m}^{2} \mathrm{SC}$ \\
\hline 1 to 28 & Thalidomide & 100 to $200 \mathrm{mg}$ ORAL \\
\hline
\end{tabular}

Quadro 23. VCD. ${ }^{37}$

\begin{tabular}{|l|l|l|}
\hline DIA & DROGA & DOSE \\
\hline 1 & Ciclofosfamida & $250 \mathrm{mg} / \mathrm{m} 2$ IV OU ORAL \\
& Dexametasona & $40 \mathrm{mg}$ IV OU ORAL \\
& Bortezomibe & $1,5 \mathrm{mg} / \mathrm{m}^{2}$ SC \\
\hline 8 & Ciclofosfamida & $250 \mathrm{mg} / \mathrm{m} 2$ IV OU ORAL \\
& Dexametasona & $40 \mathrm{mg}$ IV OU ORAL \\
& Bortezomibe & $1,5 \mathrm{mg} / \mathrm{m}^{2}$ SC \\
\hline 15 & Ciclofosfamida & $250 \mathrm{mg} / \mathrm{m} 2$ IV OU ORAL \\
& Dexametasona & $40 \mathrm{mg}$ IV OU ORAL \\
& Bortezomibe & $1,5 \mathrm{mg} / \mathrm{m}^{2}$ SC \\
\hline 28 & Dexametasona & $40 \mathrm{mg}$ IV OU ORAL \\
& Bortezomibe & $1,5 \mathrm{mg} / \mathrm{m}^{2}$ SC \\
\hline
\end{tabular}

Quadro 24. VD.

\begin{tabular}{|l|l|l|}
\hline DIA & DROGA & DOSE \\
\hline 1 & Dexametasona & $40 \mathrm{mg}$ IV OU ORAL \\
& Bortezomibe & $1,5 \mathrm{mg} / \mathrm{m}^{2}$ SC \\
\hline 8 & Dexametasona & $40 \mathrm{mg} \mathrm{IV} \mathrm{OU} \mathrm{ORAL}$ \\
& Bortezomibe & $1,5 \mathrm{mg} / \mathrm{m}^{2}$ SC \\
\hline 15 & Dexametasona & $40 \mathrm{mg} \mathrm{IV} \mathrm{OU} \mathrm{ORAL}$ \\
& Bortezomibe & $1,5 \mathrm{mg} / \mathrm{m}^{2}$ SC \\
\hline 28 & Dexametasona & $40 \mathrm{mg} \mathrm{IV} \mathrm{OU} \mathrm{ORAL}$ \\
& Bortezomibe & $1,5 \mathrm{mg} / \mathrm{m}^{2} \mathrm{SC}$ \\
\hline
\end{tabular}


Quadro 25. CD.

\begin{tabular}{|l|l|l|}
\hline DIA & DROGA & DOSE \\
\hline 1 & Ciclofosfamida & $250 \mathrm{mg} / \mathrm{m}^{2}$ IV OU ORAL \\
& Dexametasona & $40 \mathrm{mg}$ IV OU ORAL \\
\hline 8 & Ciclofosfamida & $250 \mathrm{mg} / \mathrm{m}^{2}$ IV OU ORAL \\
& Dexametasona & $40 \mathrm{mg}$ IV OU ORAL \\
\hline 15 & Ciclofosfamida & $250 \mathrm{mg} / \mathrm{m}^{2}$ IV OU ORAL \\
& Dexametasona & $40 \mathrm{mg}$ IV OU ORAL \\
\hline 28 & Dexametasona & $40 \mathrm{mg}$ IV OU ORAL \\
\hline
\end{tabular}

Quadro 26. CARFILZOMIBE + DEXAMETASONA(Endeavour). ${ }^{38}$

\begin{tabular}{|l|l|l|}
\hline DIA & DROGA & DOSE \\
\hline 1 & $\begin{array}{l}\text { Carfilzomibe } \\
\text { Dexametasona }\end{array}$ & $\begin{array}{l}56 \mathrm{mg} / \mathrm{m}^{2} \\
40 \mathrm{mg}\end{array}$ \\
\hline 2 & Carfilzomibe & $56 \mathrm{mg} / \mathrm{m}^{2}$ \\
& Dexametasona & $40 \mathrm{mg}$ \\
\hline 8 & Carfilzomibe & $56 \mathrm{mg} / \mathrm{m}^{2}$ \\
& Dexametasona & $40 \mathrm{mg}$ \\
\hline 9 & Carfilzomibe & $56 \mathrm{mg} / \mathrm{m}^{2}$ \\
& Dexametasona & $40 \mathrm{mg}$ \\
\hline
\end{tabular}

Obs.: A dose inicial de CARFILZOMIBE nos dias 1 e 2 do primeiro ciclo deve ser de $20 \mathrm{mg} / \mathrm{m}^{2}$, a seguir, aumentada para $56 \mathrm{mg} / \mathrm{m}^{2}$ a partir do dia 8 do primeiro ciclo.

Continua.

\section{REFERÊNCIAS}

1. Palumbo A. Anderson K. Multiple myeloma. N Engl J Med. 2011;364(11):1046-60

2. Rajkumar SV. Myeloma today: disease definitions and treatment advances. Am J Hematol. 2016:91:90-100.

3. Kristinsson SY, Landgren O, Dickman PH, Derolf AR, Bjorkholm M. Patterns of survival in multiple myeloma: a population-based study of patients diagnosed in Sweden from 1973 to 2003. J Clin Oncol. 2007;25:1993-9.

4. Brenner H, Gondos A, Pulte D. Recent major improvememt in long-term survival of younger patients with multiple myeloma. Blood. 2008;111:2521-6.

5. Kumar SK, Rajkumar SV, Dispethnzieri A, Lacy MQ, Hayman SR, Buadi FK, et al. Improved survival in multiple myeloma and the impact of novel therapies. Blood. 2008;111:2516-20.

6. Kumar SK, Dispenzieri A, Lacy MQ, Gertz MA, Buadi FK, Pandey $\mathrm{S}$, et al. Continued improvement in survival in multiple myeloma: changes in early mortality and outcomes in older patients. Leukemia. 2014;28(5):1122-8.

7. Hungria V, Maiolino A, Martinez G. Colleoni G, Coelho E, Rocha

L. Multiple myeloma in Brazil: clinical and demographic features
Conclusão.

Quadro 26. CARFILZOMIBE + DEXAMETASONA (Endeavour). ${ }^{38}$

\begin{tabular}{|l|l|l|}
\hline DIA & DROGA & DOSE \\
\hline 15 & Carfilzomibe & $56 \mathrm{mg} / \mathrm{m}^{2}$ \\
& Dexametasona & $40 \mathrm{mg}$ \\
\hline 16 & Carfilzomibe & $56 \mathrm{mg} / \mathrm{m}^{2}$ \\
& Dexametasona & $40 \mathrm{mg}$ \\
\hline
\end{tabular}

Obs.: A dose inicial de CARFILZOMIBE nos dias 1 e 2 do primeiro ciclo deve ser de $20 \mathrm{mg} / \mathrm{m}^{2}$, a seguir, aumentada para $56 \mathrm{mg} / \mathrm{m}^{2}$ a partir do dia 8 do primeiro ciclo.

\section{TRATAMENTO DA DOENÇA ÓSSEA ${ }^{39}$}

1. Indicado para todos os pacientes portadores de Mieloma Múltiplo sintomático que tenham ou não lesões osteolíticas detectadas.

2. Avaliação e tratamento odontológico prévios bem como educação para higiene oral adequada são necessários para prevenção de osteonecrose de mandíbula.

3. Manter por 18 a 24 meses a partir do diagnóstico.

4. Reiniciar por ocasião da recaída.

5. Necessário ajuste de dose em caso de disfunção renal.

6. Contraindicado quando Clearance de Creatinina $<30 \mathrm{ml}$.

7. Utilizar PAMIDRONATO OU ÁCIDO ZOLEDRÔNICO.

and the utility of ISS in patients, mostly with advanced disease. Haematologica. 2006;91(suppl 1):96.

8. Souza JH, Silva HF, Costa LB, Costa CM. Mieloma múltiplo: perfil epidemiológico dos pacientes acompanhados no Serviço de Hematologia do Hospital Universitário Walter Cantídio da Universidade Federal do Ceará. São Paulo: Congresso Brasileiro de Hematologia; 2008.

9. Rajkumar SV, Dimopoulos MA, Palumbo A, Blade J, Merlini G, Mateos MV, et al. International Myeloma Working Group Updated Criteria for the Diagnosis of Multiple Myeloma. Lancet Oncol. 2014; 15:e538-48.

10. Durie BG, Salmon SE. A clinical staging system for multiple myeloma. Correlation of measured myeloma cell mass with presenting clinical features, response to treatment and survival. Cancer. 1975;36(3):842-54.

11. Greipp PR, San Miguel J, Durie BG, Crowley JJ, Barlogie B, Bladé J, et al. International staging system for multiple myeloma. $\mathrm{J}$ Clin Oncol. 2005;23(15):3412-20. Erratum in J Clin Oncol. 2005;23(25):6281.

12. Palumbo A, Avet-Loiseau H, Oliva S, Oliva S, Lokhorst HM, 
Goldschmidt $\mathrm{H}$, et al. Revised international staging system for multiple myeloma: a report from International Myeloma Working Group. J Clin Oncol. 2015;33:2863.

13. Durie BG, Harousseau JL, Miguel JS, Bladé J, Barlogie B, Anderson K, et al. International uniform response criteria for multiple myeloma. Leukemia. 2006;20:1467-73.

14. Morgan GJ, Davies FE, Gregory WM, Bell SE, SzubertAJ, Navarro Coy N, et al. Cyclophosphamide, thalidomide and dexamethasone as induction therapy for newly siagnosed multiple myeloma patients destined for autologous stem-cell transplantation: MRC Myeloma IX randomized trial results. Haematologica. 2012;97(3):442- 50.

15. Davies FE, Wu P, Jenner M, Srikanth M, Saso R, Morgan GJ. The combination of cyclophosphamide, velcade and dexamethasone induces high response rates with comparable toxicity to velcade alone and velcade plus dexamethasone. Haematologica. 2007;92:1149-50

16. Reeder CB, Reece DE, Kukreti V, Chen C, Trudel S, Hentz J, et al. Cyclophosphamide, bortezomib and dexamethasone (CyBorD) induction for newly diagnosed multiple myeloma: high response rates in a phase II clinical trial. Leukemia. 2009;23(7):1337-41.

17. Jackson GH, Davies FE, Charlotte P, David A, Alina S, Corinne $\mathrm{C}$, et al. Response adapted induction treatment improves outcomes for myeloma patients; results of the phase III Myeloma XI Study. Blood. 2016;128(22):244.

18. Moreau P, Pylypenko H, Grosicki S, Karamanesht I, Leleu X, Grishunina M, et al. Subcutaneous versus intravenous administration of bortezomib in patients with relapsed multiple myeloma: a randomised, phase 3, non-inferiority study. Lancet Oncol. 2011;12:431-40.

19. Dimopolous MA, Sonneveld P, Leung N, Merlini G, Ludwig $\mathrm{H}$, Kastritis $\mathrm{E}$, et al. International Myeloma Working Group Recommendations for the diagnosis and management of myelomarelated renal impairment. J Clin Oncol. 2016;34(13):1544-57.

20. Zhu W, Chen W. Bortezomib-based treatment for multiple myeloma patients with renal impairment. A systematic review and meta-analysis of observational studies. Medicine. 2016;95:46(e5202).

21. Palumbo A, Bringhen S, Caravita T, Merla E, Capparella V, Callea V, et al. Oral melphalan and prednisone chemotherapy plus thalidomide compared with melphalan and prednisone alone in elderly patients with multiple myeloma: randomised controlled trial. Lancet. 2006;367(9513):825-31.

22. Estudo fase III de Revlimid ${ }^{\circledR}$ (lenalidomida) em Mieloma Múltiplo recém-diagnosticado FIRST, MM-020,IFM-0701): bula profissional [Internet]. Vargem Grande Paulista: Celgene; 2019 [acesso em: 15 out 2018]. Disponível em: https://media.celgene. com/content/uploads/sites/19/Revlimid_Bula_Profissional.pdf

23. Morgan GJ, Davies FE, Gregory WM, Russell NH, Bell SE, Szubert AJ, et al. Cyclophosphamide, thalidomide and dexamethasone (CTD) as initial therapy for patients with multiple myeloma unsuitable for autologous transplantation. Blood. 2011;118(5):1231-8.

24. Attal M, Harousseau JL, Leyvraz S, Doyen C, Hulin C, Benboubker $\mathrm{L}$, et al. Maintenance therapy with thalidomide improves survival in patients with multiple myeloma. Blood. 2006;108(10):3289-94.
25. Morgan GJ, Gregory WM, Davies FE, Bell SE, Szubert AJ, Brown JM, et al. The role of maintenance thalidomide therapy in multiple myeloma: MRC Myeloma IX results and meta-analysis. Blood. 2012;119(1):7-15.

26. Maiolino A, Hungria VT, Garnica M, Oliveira-Duarte G, Oliveira LC, Mercante DR, et al. Thalidomide plus dexamethasone as a maintenance therapy after autologous stem cell transplantation improves progression-free survival in multiple myeloma. Am J Hematol. 2012;87(10):948-52.

27. McCarthy PL, Holstein SA, Petrucci MT, Richardson PG, Hulin $\mathrm{C}$, Tosi $\mathrm{P}$, et al. Lenalidomide maintenance after autologous stemcell transplantation in newly diagnosed multiple myeloma: a metaanalysis. J Clin ONcol. 2017;35:3279.

28. Palumbo A, Chanan-Khan A, Weisel K, Ajay K, Tamas M, Meral B, et al. Daratumumab, Bortezomibe and Dexamethasone for multiple myeloma. N Engl J Med. 2016;375:754-6.

29. Dimopoulos MA, Oriol A, Nahi H, Jesus SM, Nizar J, Saad Z, et al. Daratumumab, Lenalidomide and Dexamethasone for multiple myeloma. N Engl J Med. 2016;375:1319-31.

30. Stewart AK, Rajkumar V, Dimopoulos MA, Masszi T, Špička I, Oriol A, et al. Carfilzomib, Lenalidomide and Dexamethasone for relapsed multiple myeloma. N Engl J Med. 2015;372:14252 .

31. Dimopoulos MA, Stewart AK, Masszi T, Špička I, Oriol A, Hájek R, et al. Carfilzomib, Lenalidomide and Dexamethasone in patients with relapsed multiple myeloma categorised by age: secondary analysis from the phase 3 ASPIRE Study. Brit J Haematol. 2017; 177:404-13.

32. Moreau P, Masszi T, Grzasko N, Nizar J, Markus H, Ludek P, et al. Oral Ixazomib, Lenalidomide and Dexamethasone for multiple myeloma. N Engl J Med. 2016;374:1621-34.

33. Sidra G, Williams CD, NH Russell, S Zaman, B Myers, JL Byrne. Combination chemotherapy with cyclophosphamide, thalidomide and dexamethasone for patients with refractory, newly diagnosed or relapsed myeloma. Haematologica. 2006;91:862-3.

34. Lonial S, Dimopoulos M, Palumbo A, White D, Grosicki S, Spicka I, et al. Elotuzumab therapy for relapsed or refractory multiple myeloma. New J Med. 2015;373:621-31.

35. García-Sanz R, González-Porras JR, Hernández JM, PoloZarzuela M, Sureda A, Barrenetxea C, et al. The oral combination of thalidomide, cyclophosphamide and dexamethasone (ThaCyDex) is effective in relapsed/refractory multiple myeloma. Leukemia. 2004;18(4):856-63.

36. Davies FA, Wu P, Jenner M, Srikanth M, Saso R, Morgan GJ. The Combination of Cyclophosphamide, Velcade and/dexamethasone (CVD) induces high response rates with comparable toxicity to Velcade alone (V) and Velcade plus Dexamethasone (VD). Haematologica. 2007;92:1149-50.

37. Pineda-Roman M, Zangari M, van Rhee F, Anaissie E, Szymonifka J, Hoering A, et al. VTD combination therapy with bortezomib-thalidomide dexamethasone is highly effective in advanced and refractory multipke myeloma. Leukemia. 2008;22(7):1419-27. 
38. Dimopoulos MA, Moreau P, Palumbo A, Joshua D, Pour L, Hájek R, et al. Carfilzomib and Dexamethasone versus Bortezomib and Dexamethasone for Patients with relapsed or refractory multiple myeloma (ENDEAVOR): a randomised phase 3, open-label, multicentre study. Lancet Oncol. 2016;17(1):27-38.
39. Terpos E, Morgan G, Dimopoulos MA, Drake MT, Lentzsch S, Raje N, et al. International Myeloma Working Group recomendations for the treatment of multiple myeloma - related bone disease. J Clin Oncol. 2013;31:2347-57.

\section{Como citar:}

Sousa JH, Silva HF, Gurgel LA, Leitão JP, Araújo BS, Barroso KS, et al. Protocolo de tratamento do mieloma múltiplo serviço de hematologia Hospital Universitário Walter Cantídio, abril - 2019. Rev Med UFC. 2020 jan-mar;60(1):63-71. 